SCIENTIFIC REPORT

\title{
Association between choroidal pigmentation and posterior uveal melanoma in a white population
}

\author{
J W Harbour, M A Brantley Jr, H Hollingsworth, M Gordon
}

Br J Ophthalmol 2004;88:39-43

\begin{abstract}
Background/aims: It is well known that light skin pigmentation is a risk factor for cutaneous melanoma. The aim of this study was to investigate the analogous association between choroidal pigmentation and posterior uveal melanoma.

Methods: Cross sectional study of 65 consecutive patients diagnosed with posterior uveal melanoma (melanoma group) and 218 consecutive patients referred for general retinal evaluation (control group). All patients were white. A clinical grading system for estimating choroidal pigmentation was developed and histologically validated in seven patients. Results: Melanoma patients with light iris colour were significantly more likely to have darker choroidal pigmentation than controls $(p=0.005)$. Darker choroidal pigmentation was associated histologically with increased density of choroidal melanocytes ( $p=0.005)$.

Conclusions: Increased choroidal pigmentation, as a result of an increase in the density of pigmented choroidal melanocytes, is not protective but may actually be a risk factor for the development of posterior uveal melanoma in white patients. This finding may have implications for understanding the pathogenesis of uveal melanoma.
\end{abstract}

$\mathrm{U}$ veal melanoma is the most common primary eye cancer, and it is the most common site of melanoma besides the skin. ${ }^{12}$ The environmental and genetic risk factors for uveal melanoma remain less clear than for cutaneous melanoma, where there is now strong evidence that UV irradiation plays an important role. ${ }^{3}$ Several studies have reported associations between uveal melanoma and increased UV exposure, sensitivity to UV light, ancestry from northern latitudes, light skin colour, residence at lower latitudes, sunlamp use, and a history of intense sunlight exposure. ${ }^{4-6}$ However, other studies have failed to confirm an association between uveal melanoma and UV exposure. ${ }^{12}$

Several investigators have also reported an association between uveal melanoma and light iris colour. ${ }^{5-7}$ Based on the assumption that light iris colour may allow more UV light to reach the posterior segment, these studies have generally been interpreted as supporting an aetiologic role for UV exposure in uveal melanoma. However, it remains unproven whether significant levels of UV irradiation reach the posterior segment in adult human eyes, irrespective of iris colour. $^{8}$

Choroidal pigmentation is largely caused by melanin pigment within choroidal melanocytes. Individuals with dark racial pigmentation generally have very heavy choroidal pigmentation and are at extremely low risk of uveal melanoma. Among white people, choroidal pigmentation varies from heavy to very light (for example, "blonde fundus"). As whites are the racial group at highest risk for posterior uveal melanoma, we performed a study to determine whether light choroidal pigmentation may be a risk factor for uveal melanoma, as light skin pigmentation is a risk factor cutaneous melanoma. We developed a clinical grading system for estimating the degree of choroidal pigmentation, and we confirmed the histologic validity of this grading system in a clinicopathologic correlative study. We then used this grading system in a cross sectional study of patients with posterior uveal melanoma and control patients.

\section{SUBJECTS AND METHODS}

The subjects consisted of a consecutive series of patients examined by one of the authors (JWH) at the Barnes Retina Institute between February 1998 and October 1999 and who met inclusion criteria into one of two diagnostic groups: posterior uveal melanoma group or control group. The following clinical features were recorded for each patient: age, gender, race, iris colour, and choroidal pigmentation (see below). The study was conducted in accordance with a protocol approved by the institutional Human Studies Committee.

\section{Melanoma group}

All patients in the melanoma group were examined, diagnosed, and treated for posterior uveal melanoma by one physician (JWH). All patients included in this group were found to have an elevated tumour involving the choroid and/or ciliary body with clinical and ultrasonographic features consistent with uveal melanoma, including low to medium internal reflectivity and a thickness greater than $3 \mathrm{~mm}$ by standardised A-scan ultrasonography (or documented growth if initial thickness less than $3 \mathrm{~mm}$ ). Exclusion criteria included clinical or ultrasound characteristics that are atypical for melanoma, such as (1) multiple tumours, (2) thickness less than $3 \mathrm{~mm}$ in the absence of documented growth, and (3) high or irregular internal reflectivity on A-scan ultrasonography. Patients were also excluded if the ocular media were opacified, precluding adequate examination of the anterior and posterior segments. For each melanoma the following features were noted: basal tumour dimensions (ophthalmoscopic estimate), tumour thickness (A-scan), tumour location by quadrant (superotemporal, superonasal, inferotemporal, inferonasal) and relationship to the equator (anterior versus posterior).

\section{Control group}

The control group consisted of a consecutive series of patients that were referred to JWH for general retinal evaluation. Exclusion criteria included: (1) referral for a suspected uveal neoplasm, (2) media opacity that precluded examination of the iris and choroid of both eyes, (3) iris heterochromia, or (4) history of disorders that could alter iris appearance or colour (for example, iris neovascularisation, Fuchs' heterochromic cyclitis, iris nodules, iridocorneal endothelial syndrome). As all patients in the melanoma group were white, 
only white patients were included in the control group. In an attempt to obtain a control group matched for age with melanoma patients, control patients under 20 years old were excluded. Patients in the control group diagnosed with macular degeneration (70 patients), diabetic retinopathy (36 patients), posterior vitreous separation (17 patients), retinal vascular occlusion (15 patients), cystoid macular oedema (10 patients), retinal detachment (nine patients), epiretinal membrane (nine patients), myopia (nine patients), lattice degeneration (six patients), macular hole (six patients), retinal tear (four patients), and a variety of other non-neoplastic conditions (one to two patients each).

\section{Measurement of iris colour and choroidal pigmentation}

In order to increase the statistical power of analysis, iris colour was graded in a binomial fashion as either light (including blue, green, and grey irides) or dark (brown irides). A grading system was developed for estimating choroidal pigmentation on clinical examination. Three grades of choroidal pigmentation were established that could be distinguished on indirect ophthalmoscopy: light (large choroidal vessels appear darker than the surrounding choroid due to minimal pigmentation between vessels), medium (large choroidal vessels are difficult to distinguish from the surrounding choroid due to patchy or incomplete choroidal pigmentation, and dark (large choroidal vessels appear lighter than, and stand out in relief against, the surrounding dark choroidal pigment between the vessels). As choroidal pigmentation is not always evenly distributed throughout the fundus and is often darker in the macula, grading of choroidal pigmentation was assessed at the fundus midperiphery, just outside the temporal vascular arcades and nasal to the optic disc.

\section{Clinical-histological analysis of choroidal pigmentation}

Clinical-histological correlation was performed in seven eyes that became available for histological evaluation after clinical grading. Eyes were excluded if there was a history of plaque radiotherapy or if satisfactory histological sections of the fundus midperiphery were not available. Each eye was fixed in formalin, embedded in paraffin, sectioned and stained with haematoxylin-eosin or prepared for immunohistochemistry with HMB-45 antibody (Dako, Glostrup, Denmark). Immunohistochemistry was performed using the streptavidin-biotin method with the Vector ABC Elite kit (Vector Laboratories, Inc, Burlingame, CA, USA). Nuclear fast red was used for counterstain. Four micron sections were obtained, deparaffinised, rehydrated with ethanol, and treated with $0.3 \%$ hydrogen peroxide and methanol to inhibit endogenous peroxidase activity. Heat induced antigen retrieval was performed using microwave treatment in citrate buffer for 15 minutes. Primary HMB-45 antibody was applied at $4^{\circ} \mathrm{C}$ overnight. Sections of each eye were examined microscopically in a masked fashion. Choroidal melanocytes were counted at $100 \times$ magnification under oil immersion in at least four different fields in the fundus midperiphery. The number of choroidal melanocytes that were present within a high power field was determined by counting melanocyte nuclei.

\section{Statistical methods}

To compare the distribution of ages of the two groups of patients, a single factor analysis of variance was performed. $\chi^{2}$ tests of independence were performed to analyse the association between (1) gender and group membership, (2) iris colour and group membership, (3) iris colour and tumour location, (4) choroidal pigmentation grade and group membership, and (5) choroidal pigmentation grade and tumour location. Pearson's correlation coefficient was used to analyse the association between choroidal pigmentation grade and number of choroidal melanocytes. Statistical significance was assumed for $\mathrm{p}<0.05$.

\section{RESULTS \\ Description of patients}

A series of 283 consecutive patients meeting inclusion criteria were placed into the melanoma group (65 patients) or control group (218 patients). Table 1 summarises the clinical information. There was a significant difference in the mean age of the control group (68.4 years; range, 20-95 years) versus the melanoma group (62.4 years; range, $24-86$ years) $(p=0.003)$. There was a non-significant excess of males in the melanoma group $(63.1 \%)$ compared with the control group $(41.7 \%)(p=0.996)$. None of the patients in either group had ocular melanocytosis, familial cutaneous dysplastic nevi, or melanomas.

\section{Iris colour}

The proportion of patients with light iris colour in the melanoma group was $83.0 \%$ and in the control group was $72.0 \%(p=0.12)$. There was no relation between iris colour and tumour location $(\mathrm{p}=0.691)$.

\section{Choroidal pigmentation}

We developed a clinical grading system based on indirect ophthalmoscopy for estimating the degree of choroidal pigmentation (fig 1). Choroidal pigmentation was dark in $58.4 \%$ of the melanoma group and $42.6 \%$ of the control group; this difference did not achieve statistical significance $(p=0.06)$. As all but one patient with dark iris colour had medium or dark choroidal pigmentation, we then stratified patients by iris colour to allow the subgroup with light iris colour to be evaluated (table 2). Among patients with light iris colour, the prevalence of dark choroidal pigmentation was $54.7 \%$ in the melanoma group and $30.4 \%$ in the control group. This association between dark choroidal pigmentation and melanoma was significant $(p=0.005)$. There was no

Table 1 Patient and tumour characteristics

\begin{tabular}{|c|c|c|}
\hline Patient and tumour characteristics & Melanoma & Control \\
\hline Number of patients & 65 & 218 \\
\hline Mean age (years) & 62.6 & 68.4 \\
\hline \multicolumn{3}{|l|}{ Sex } \\
\hline Male & $42(64.6 \%)$ & $91(41.7 \%)$ \\
\hline Female & $23(35.4 \%)$ & $127(58.3 \%)$ \\
\hline \multicolumn{3}{|l|}{ Melanoma size (mean) } \\
\hline Largest basal dimension & $12.9 \mathrm{~mm}$ & - \\
\hline Smallest basal dimension & $10.2 \mathrm{~mm}$ & - \\
\hline Thickness & $6.04 \mathrm{~mm}$ & - \\
\hline \multicolumn{3}{|l|}{ Melanoma location } \\
\hline Superonasal & $10(15.4 \%)$ & - \\
\hline Superotemporal & $23(35.4 \%)$ & - \\
\hline Inferonasal & $11(16.9 \%)$ & - \\
\hline Inferotemporal & $21(32.3 \%)$ & - \\
\hline \multicolumn{3}{|l|}{ Melanoma location relative to equator } \\
\hline Anterior & $21(32.3 \%)$ & - \\
\hline Posterior & $44(67.7 \%)$ & - \\
\hline \multicolumn{3}{|l|}{ Iris colour } \\
\hline Light & $53(81.5 \%)$ & $158(72.5 \%)$ \\
\hline Dark & $12(18.5 \%)$ & $60(27.5 \%)$ \\
\hline \multicolumn{3}{|l|}{ Choroidal pigmentation } \\
\hline Light & 10 (15.4\%) & 37 (17.0\%) \\
\hline Medium & $17(26.1 \%)$ & 88 (40.4\%) \\
\hline Dark & 38 (58.4\%) & $93(42.6 \%)$ \\
\hline
\end{tabular}



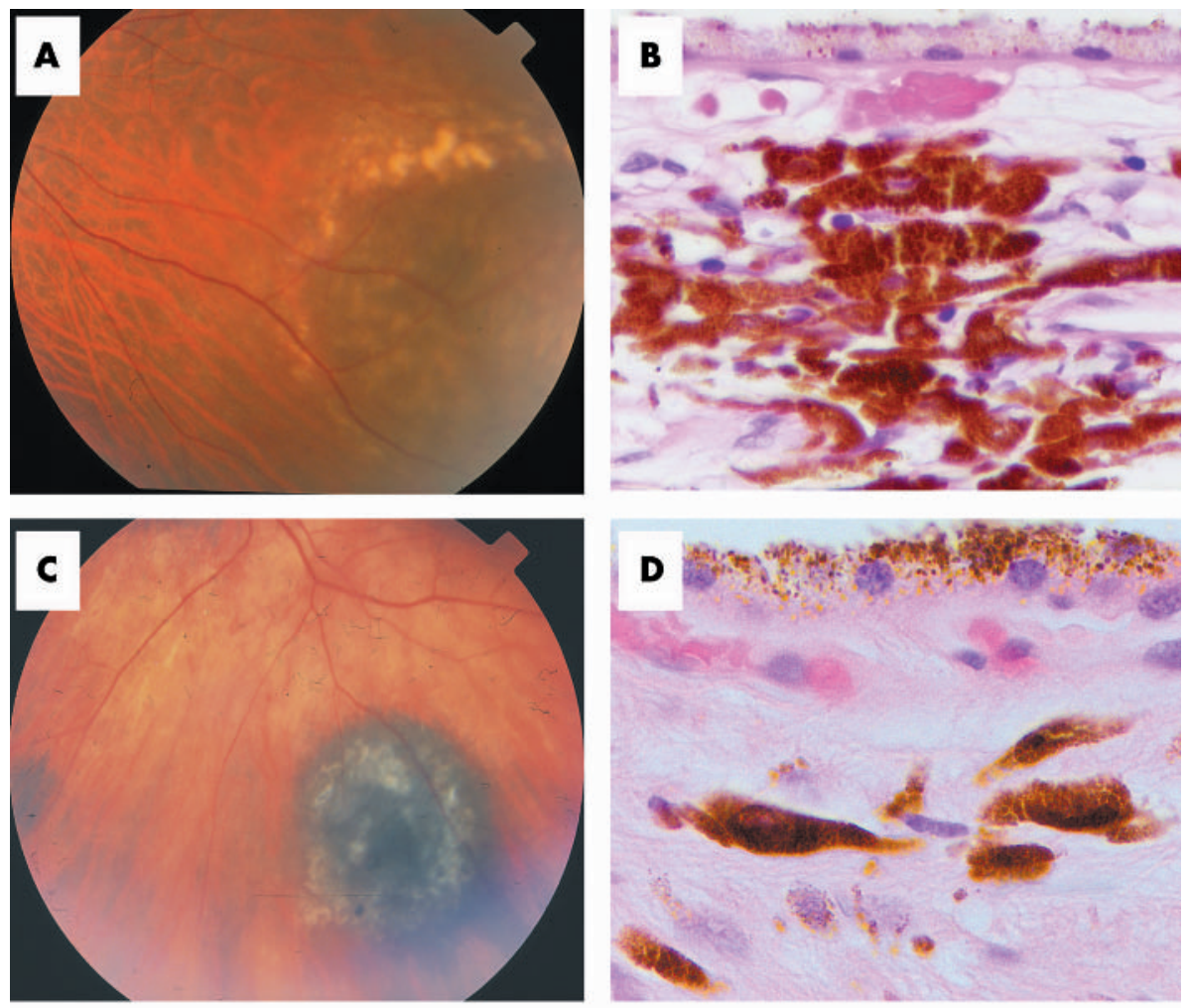

Figure 1 Clinical-histological correlation of choroidal pigmentation grading system. (A) Dark choroidal pigmentation (large choroidal vessels appear lighter than choroidal pigment). (B) Histologic correlate of dark choroidal pigmentation (dense population of large pigmented melanocytes). (C) Medium choroidal pigmentation (large choroidal vessels are difficult to distinguish from choroidal pigment). (D) Histologic correlate of medium choroidal pigmentation (mixture of large and small melanocytes). (E) Light choroidal pigmentation (large choroidal vessels appear darker than choroidal pigment). (F) Histologic correlate of light choroidal pigmentation (sparse population of lightly pigmented melanocytes).
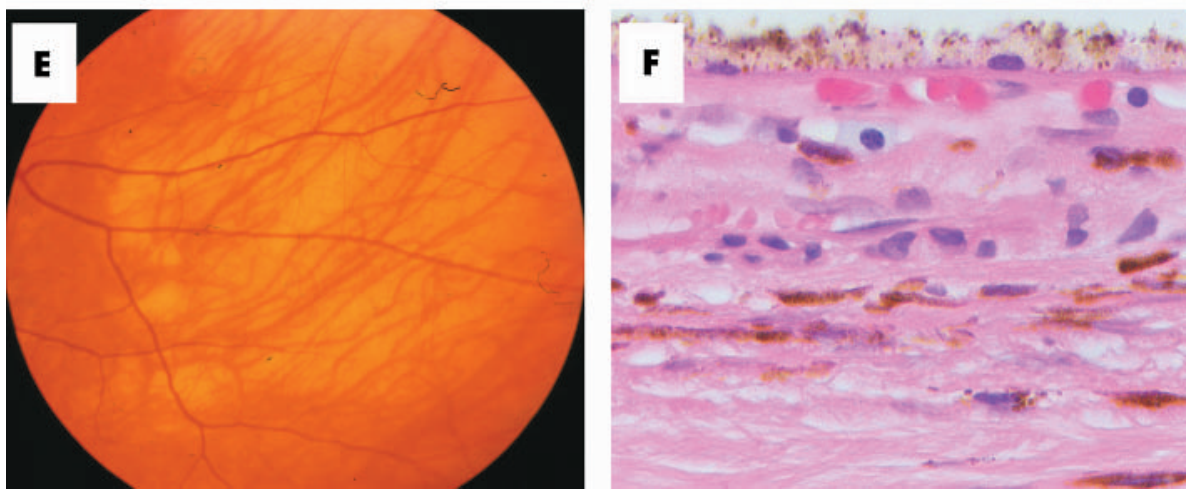

association between choroidal pigmentation and tumour location $(\mathrm{p}=0.231)$.

\section{Clinical-histological correlation of choroidal pigmentation}

Nine eyes from the melanoma group subsequently required enucleation and became available for histological examination. Two eyes were excluded due to prior radiotherapy (one

Table 2 Stratification of iris and choroidal pigmentation by patient group

\begin{tabular}{llccr}
\hline & & \multicolumn{3}{c}{ Choroidal pigmentation } \\
\cline { 4 - 5 } Patient group & $\begin{array}{l}\text { Iris } \\
\text { colour }\end{array}$ & Light & Medium & Dark \\
\hline Melanoma $(\mathrm{n}=65)$ & & $10(15 \%)$ & $14(22 \%)$ & $29(45 \%)$ \\
& Light & $10(15 \%)$ & $3(4 \%)$ & $9(14 \%)$ \\
Control $(\mathrm{n}=218)$ & Dark & $0(0 \%)$ & & \\
& Light & $36(17 \%)$ & $74(34 \%)$ & $48(22 \%)$ \\
& Dark & $1(<1 \%)$ & $14(6 \%)$ & $45(21 \%)$ \\
\hline
\end{tabular}

eye) or lack of histologic sections satisfactory for choroidal examination (one eye). In the remaining seven cases, the clinical grade for choroidal pigmentation had been light (one eye), medium (four eyes), or dark (two eyes). Histological analysis of sections stained with haematoxylin-eosin or immunostained for HMB-45 was performed in a masked fashion, and the morphology and number of choroidal melanocytes were determined. The mean (SD) number of choroidal melanocytes per high power field was 31.2 (3.4) (light choroidal pigmentation), 66 (10.8) (medium choroidal pigmentation), and 118 (24.6) (dark choroidal pigmentation), and the correlation between choroidal pigmentation grade and the density of choroidal melanocytes was highly significant $(p=0.002)$ (fig 2$)$. Immunostaining for HMB-45 showed that only a small fraction $(<1 \%)$ of melanocytes were unpigmented in each case. Pigment within retinal pigment epithelial cells was similar among all three pigmentation groups. Darker choroidal pigmentation also appeared to correspond to increased size and number of cytoplasmic pigment granules within melanocytes and increase in overall cellularity of the choroid (fig 1), although these parameters were not quantified. 


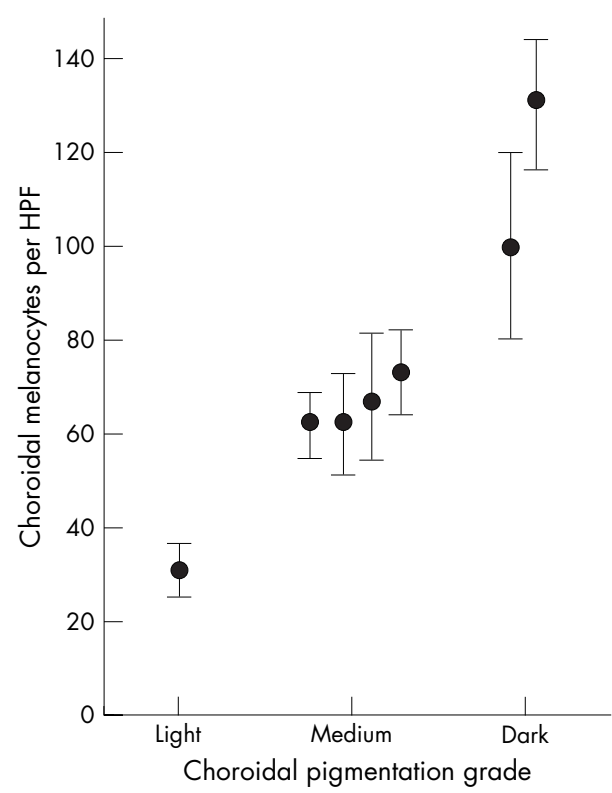

Figure 2 Graph showing strong correlation between clinical choroidal pigmentation grade ( $x$ axis) and the number of choroidal melanocytes per $100 \times$ high power field (y axis). Error bars indicate one standard deviation.

\section{DISCUSSION}

We have developed a histologically validated clinical grading system for estimating the degree of choroidal pigmentation, and we used this system in a cross sectional study to investigate the relation between choroidal pigmentation and posterior uveal melanoma. Based on the known association between light skin pigmentation and cutaneous melanoma, and the rarity of uveal melanoma among individuals with dark racial pigmentation, we anticipated that light choroidal pigmentation might be a risk factor for posterior uveal melanoma. However, we were surprised to find a significant association between darker choroidal pigmentation and posterior uveal melanoma $(p=0.005)$ in white patients with light iris colour. We conclude that dark choroidal pigmentation is not protective but may be a risk factor for posterior uveal melanoma among white people with light iris colour.

One potential explanation for this finding is that whites with dark choroidal pigmentation may be at higher risk of oxidative DNA damage in choroidal melanocytes, compared with whites with light choroidal pigmentation. Melanin is a photoreactive compound and can paradoxically act as a photosensitiser, as well as a photoprotector." Increased intracellular melanin can increase the susceptibility of melanocytes to UV-induced DNA damage by the production of reactive oxygen species. ${ }^{10}$ Consistent with this model, heavily pigmented melanocytes have been shown to contain higher levels of UV-induced DNA damage than lightly pigmented melanocytes. ${ }^{11}$ Most individuals with dark racial pigmentation have choroidal pigmentation that is significantly heavier than the "dark" choroidal pigmentation of whites in this study. Thus, the photoprotective effects of melanin may greatly outweigh the photosensitising effects at the vastly higher levels of choroidal melanin in these individuals. Further, dark pigmented individuals produce more eumelanin, a form of melanin that has a much greater photoprotective effect than pheomelanin, which is the form of melanin that is more commonly found in fair skinned individuals. ${ }^{12}$ Studies of melanin composition in human choroidal melanocytes are needed to determine whether choroidal melanin composition is an important risk factor for uveal melanoma.

Another possible explanation for our finding is that the darker choroidal pigmentation may represent a response to chronic higher levels of UV irradiation. In support of this idea, Li et al recently reported a correlation between light iris colour and posterior uveal melanoma location. ${ }^{13}$ They demonstrated that the distribution of tumours correlated with the dose distribution of solar light on the posterior segment, and they concluded that these findings support a role for UV irradiation in the development of posterior uveal melanoma. However, other workers found a lack of correlation between melanoma location and UV light dose distribution. ${ }^{14}$ This explanation would seem to be unlikely as one would expect the iris to become more pigmented as well since it is exposed to higher levels of UV light than the choroid.

A third possibility is a simple stochastic explanation. The more melanocytes that are present in the choroid, the more likely it may be that one will undergo malignant transformation. This model does not require (but does not rule out) an aetiological role for UV exposure. This model could also explain the well recognised association between uveal melanoma and ocular melanocytosis, a condition characterised by a marked increase in the number of melanocytes throughout the uveal tract. ${ }^{15}$ However, this theory does not easily explain the paucity of uveal melanoma in darkly pigmented races. A final possibility is that this statistical association occurred by chance. Hence, these findings need to be confirmed by other investigators.

Advantages of our study design include prospective data collection by a single physician, consecutive patient entry, and histologic verification of the choroidal pigmentation grading system. Disadvantages of the study design include a control group of patients that were referred for retinal evaluation. This group may not precisely correspond to the population at risk for uveal melanoma. About one third of controls were referred for macular degeneration, which has been linked to light iris colour. However, it is unclear whether inclusion of these patients detracts from the analysis, as these patients would appear to bias the study against our finding that patients with light iris colour and darker choroidal pigmentation were at higher risk for uveal melanoma. Patient groups differed with respect to age, which may be an important determinant of melanocyte density and pigmentation. ${ }^{16}$ However, since the mean difference in age was only six years, this difference is unlikely to affect our conclusions. None of the melanoma or control patients had a history of ocular melanocytosis, atypical cutaneous nevi, or cutaneous melanoma. Hence, the results of this study may not be applicable to rare patients with melanoma predisposition syndromes.

The choroidal pigmentation grading system proved to be simple to perform using indirect ophthalmoscopy, and the results correlated strongly with histological findings. Dark choroidal pigmentation reflected an increase in density of choroidal melanocytes, and also appeared to correlate with increased size and density of cytoplasmic melanosomes. By comparison, iris colour appears to be determined primarily by the number and size of melanosomes within iris stromal melanocytes. ${ }^{17}{ }^{18}$ To our knowledge, this is the first report of a clinical-histological correlation of choroidal pigmentation.

In summary, this study has identified an unexpected association between dark choroidal pigmentation and posterior uveal melanoma in white patients with light iris colour. Although the explanation for this finding is not clear, several possible hypotheses are presented here as a basis for further investigations to determine the role of UV irradiation, iris colour, and choroidal pigmentation in the pathogenesis of uveal melanoma. 


\section{ACKNOWLEDGEMENTS}

The Biostatistics Core Facility is supported by a grant from the National Eye Institute.

\section{Authors' affiliations}

J W Harbour, M A Brantley, M Gordon, Department of Ophthalmology and Visual Sciences, Washington University, St Louis, Missouri, USA

H Hollingsworth, Programme in Occupational Therapy, Washington

University, St Louis, Missouri, USA

Correspondence to: J William Harbour, Department of Ophthalmology and Visual Sciences, Washington University School of Medicine, Box 8069, 660 S. Euclid Avenue, St Louis, MO 63110, USA;

harbour@vision.wustl.edu

Accepted for publication 2 June 2003

\section{REFERENCES}

1 Egan KM, Seddon JM, Glynn RJ, et al. Epidemiologic aspects of uveal melanoma. Surv Ophthalmol 1988;32:239-51

2 Scotto J, Fraumeni JF Jr, Lee JA. Melanomas of the eye and other noncutaneous sites: epidemiologic aspects. J Natl Cancer Inst 1976;56:489-91.

3 de Gruijl FR. Skin cancer and solar UV radiation. Eur J Cancer 1999:35:2003-9.

4 Seddon JM, Gragoudas ES, Glynn RJ, et al. Host factors, UV radiation, and risk of uveal melanoma. A case-control study. Arch Ophthalmol 1990; 108:1274-80
5 Tucker MA, Shields JA, Hartge $P$, et al. Sunlight exposure as risk factor for intraocular malignant melanoma. N Engl J Med 1985;313:789-92.

6 Holly EA, Aston DA, Ahn DK, et al. Intraocular melanoma linked to occupations and chemical exposures. Epidemiology 1996:7:55-61.

7 Gallagher RP, Elwood JM, Rootman J, et al. Risk factors for ocular melanoma: Western Canada Melanoma Study. J Natl Cancer Inst 1985;74:775-8.

8 Reme C, Reinboth J, Clausen M, et al. Light damage revisited: converging evidence, diverging views? Graefes Arch Clin Exp Ophthalmol 1996;234:2-11.

9 Hill HZ, Li W, Xin P, et al. Melanin: a two edged sword? Pigment Cell Res 1997; 10:158-61.

10 Kvam E, Tyrrell RM. The role of melanin in the induction of oxidative DNA base damage by ultraviolet A irradiation of DNA or melanoma cells. J Invest Dermatol 1999; 113:209-13.

11 Hill HZ, Hill GJ. UVA, pheomelanin and the carcinogenesis of melanoma. Pigment Cell Res 2000;13:140-4.

12 Cone RD, Lu D, Koppula S, et al. The melanocortin receptors: agonists, antagonists, and the hormonal control of pigmentation. Recent Prog Horm Res 1996;51:287-317

13 Li W, Judge H, Gragoudas ES, et al. Patterns of tumor initiation in choroidal melanoma. Cancer Res 2000:60:3757-60.

14 Schwartz LH, Ferrand R, Boelle PY, et al. Lack of correlation between the location of choroidal melanoma and ultraviolet-radiation dose distribution. Radiat Res 1997; 147:451-6.

15 Singh AD, De Potter P, Fijal BA, et al. Lifetime prevalence of uveal melanoma in white patients with oculo(dermal) melanocytosis. Ophthalmology 1998;105:195-8.

16 Nordlund JJ. The lives of pigment cells. Clin Geriatr Med 1989;5:91-108.

17 Wilkerson CL, Syed NA, Fisher MR, et al. Melanocytes and iris color. Light microscopic findings. Arch Ophthalmol 1996;114:437-42.

18 Imesch PD, Bindley CD, Khademian Z, et al. Melanocytes and iris color. Electron microscopic findings. Arch Ophthalmol 1996;114:443-7.

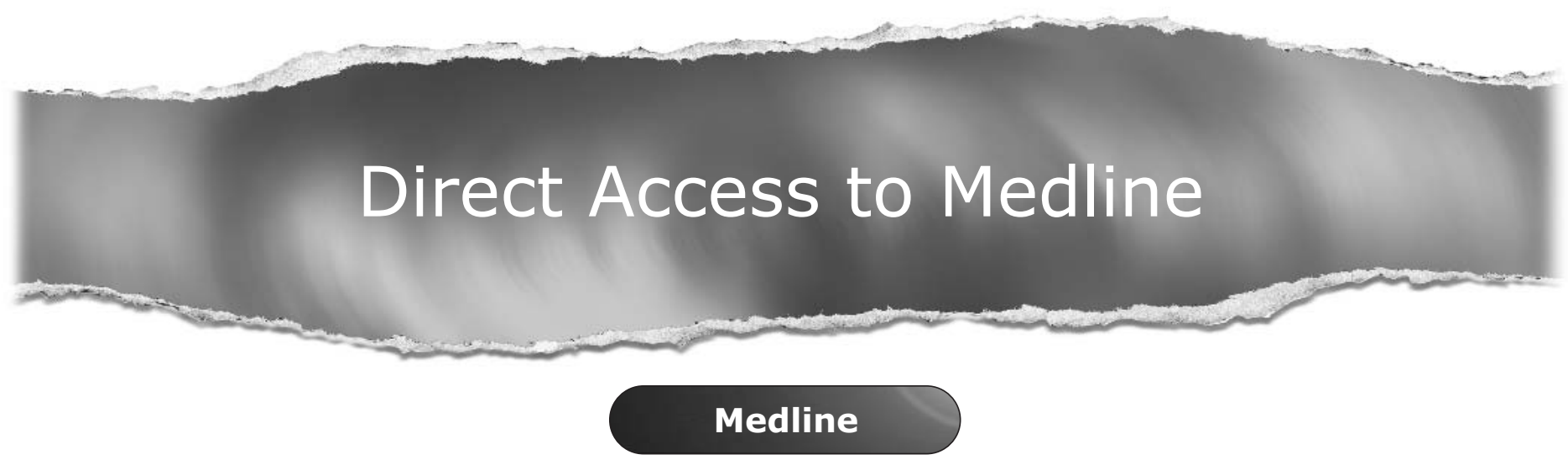

Link to Medline from the homepage and get straight into the National Library of Medicine's premier bibliographic database. Medline allows you to search across 9 million records of bibliographic citations and author abstracts from approximately 3,900 current biomedical journals.

\section{www.bjophthalmol.com}

